\title{
Framework to benchmark sustainability of biomethane supply chains: Facilitating sustainability decision making in adopting biomethane as a public transportation fuel in Western Europe
}

\author{
Tharaka Gunaratne, Stefan Dahlgren and Larsgöran Strandberg \\ Journal Article
}

\section{Tweet}

N.B.: When citing this work, cite the original article.

This is an electronic version of an article published in:

Tharaka Gunaratne, Stefan Dahlgren and Larsgöran Strandberg, Framework to benchmark sustainability of biomethane supply chains: Facilitating sustainability decision making in adopting biomethane as a public transportation fuel in Western Europe, International Journal of Green Energy, 2016. 13(8), pp.759-766.

International Journal of Green Energy is available online at informaworldTM:

http://dx.doi.org/10.1080/15435075.2016.1175352

Copyright: Taylor \& Francis: STM, Behavioural Science and Public Health Titles

http://www.tandf.co.uk/journals/default.asp

Postprint available at: Linköping University Electronic Press

http://urn.kb.se/resolve?urn=urn:nbn:se:liu:diva-133028

LINKÖPINGS

UNIVERSITET 


\title{
FRAMEWORK TO BENCHMARK SUSTAINABILITY OF BIOMETHANE SUPPLY CHAINS
}

\section{FACILITATING SUSTAINABILITY DECISION MAKING IN ADOPTING BIOMETHANE AS A PUBLIC TRANSPORTATION FUEL IN WESTERN EUROPE}

\author{
Tharaka Gunaratne (Corresponding author) ${ }^{1}$, Stefan Dahlgren ${ }^{2}$, Larsgöran Strandberg ${ }^{3}$ \\ ${ }^{1}$ WSP AB, Arenavägen 7, 121 88, Stockholm, Sweden. tharaka.gunaratne@wspgroup.se +46107228686 \\ ${ }^{2}$ WSP AB, Arenavägen 7, 121 88, Stockholm, Sweden. stefan.dahlgren@wspgroup.se \\ ${ }^{3}$ KTH Industrial Ecology, Teknkringen 34, 114 28, Stockholm, Sweden. Igs@kth.se
}

\begin{abstract}
Among various strategies nurtured to triumph energy and climate issues, adoption of cleaner sources for energy production lead in the way. In this article a framework has been presented from a systems perspective to benchmark biomethane supply chains consisting of biogas upgrading, biomethane distribution and refilling. The framework is mostly applicable in the context of Western Europe as far as biogas upgrading technologies and costs are concerned.

Even though compressed biomethane has been widely adopted as a vehicle fuel, promoting liquefied biomethane appears to be viable in many aspects. However the upgrading technologies for the latter is still in infancy stages and therefore the accuracy of performance is still not proven solidly. In contrast, venturing into the former guarantees a more secure investment with proven accuracy of results and widespread availability of technological knowhow. Distribution of compressed biomethane through gas grids appears to be a feasible option only in short distances and large scale whereas using trucks with composite swap bodies is the preferable alternative for biomethane distribution otherwise. As a whole, nurturing liquefied biomethane supply chains manifests better performance from both climate and cost perspectives while adopting compressed biomethane supply chains based on gas grids would lead to better energy intensity.
\end{abstract}

Keywords: Biomethane, Benchmarking, Climate change impact, Energy intensity, Cost effectiveness

\begin{tabular}{|c|c|}
\hline OMEN & URE \\
\hline EU & European Union \\
\hline $\mathrm{CH}_{4}$ & Methane \\
\hline $\mathrm{CO}_{2}$ & Carbon Dioxide \\
\hline CBM & Compressed Biomethane \\
\hline LBM & Liquefied Biomethane \\
\hline ICE & Investment Cost Effectiveness \\
\hline OMCE & $\begin{array}{l}\text { Operation and Maintenance } \\
\text { Effectiveness }\end{array}$ \\
\hline TCE & Total Cost Effectiveness \\
\hline $\mathrm{El}$ & Energy Intensiveness \\
\hline $\mathrm{Cl}$ & Climate Impact \\
\hline
\end{tabular}

\section{INTRODUCTION}

Transportation plays a vital role in any developed country as far as the energy mix is concerned. Thus the European Union through its 'Renewable energy directive' has made a special requirement to achieve $10 \%$ renewable energy share in the transport sector. Transportation on average accounts for more than $30 \%$ of the EU's primary energy consumption [1].

In order to be able to be combusted in a vehicle engine, biogas needs to be upgraded into a certain quality in terms of the methane $\left(\mathrm{CH}_{4}\right)$ content. The upgraded biogas is called biomethane and is readily available to be used in vehicle engines that already combust natural gas.

A substantial amount of literature is available addressing the upgrading of raw biogas to biomethane whereas literature encompassing the fields of biomethane distribution and refilling is also not scarce. A significant research gap nevertheless has been identified with relation to analysis of sustainability of alternative biomethane supply chains in their entirety. Such an analysis is deemed essential in assisting sustainability decision making in real time enterprises in biomethane systems. 
The aim of this article is therefore to present a framework for benchmarking sustainability of biomethane supply chains, consisting of three stages; biogas upgrading (biomethane production), biomethane distribution and refilling. Thereby the formulated framework is expected to assist sustainability decision making in fostering biomethane systems for transportation.

In order to achieve the aforementioned aim, a set of objectives have been formulated as follows.

1. Identify common alternatives in commercial scale for each of the three stages of biomethane supply chain

2. Assess the sustainability performance of the selected alternatives at each stage.

3. Analyze the sustainability parameters of alternative biomethane supply chains and the sensitivities of results.

\section{METHODOLOGY}

The methodology explains the process of this study with respect to the aspects; identification of commercially available alternatives at each stage of biomethane supply chain, research, calculation of sustainability indicators, results and discussion, and sensitivity analysis.

\subsection{Identification of commercially available alternatives}

\subsubsection{Biomethane Production}

The scale of biomethane production considered is from $300 \mathrm{Nm}^{3} / \mathrm{hr}$ to $3000 \mathrm{Nm}^{3} / \mathrm{hr}$ of raw gas utilization. Thereafter the production has been classified according to the two main principles of biogas upgrading (or biomethane production) at present. That is producing biomethane as compressed gas which is hereafter called as compressed biomethane (CBM) and liquefied biomethane gas which hereafter is called as liquefied biomethane (LBM). Thus two production technologies under each category have been selected as follows.

Technologies for producing CBM;

I. The water scrubber technology COMPACT ${ }^{\mathrm{TM}}$ [2]

II. The chemical scrubber technology CApure ${ }^{\mathrm{TM}}$ [3]

> Technologies for producing LBM;

I. The cryogenic technology GPP plus [4]

II. The cryogenic technology $\mathrm{CO}_{2}$ Wash ${ }^{\oplus}$ [5]

\subsubsection{Biomethane distribution}

Distribution of Biomethane is discussed with respect to two categories; distribution method and the distribution distance.

1) Classification based on the distribution method;
$>$ Distribution methods for CBM

I. Gas grid

II. Hook lift swap body trailers

a. Swap bodies made of steel

b. Swap bodies made of composite material

$>$ Distribution method for LBM

I. Liquefied biomethane trailer

2) Classification based on the distribution distance;
I. Short distance $-10 \mathrm{~km}$
II. Medium distance $-25 \mathrm{~km}$
III. Long distance $\quad-100 \mathrm{~km}$

Distribution along a distance which is in between any two of the above three levels is to be interpolated assuming a linear correlation.

\subsubsection{Biomethane refilling}

Methods for refilling biomethane into vehicles at filling stations are also classified according to the state of fuel delivered.

$>$ Refilling methods considered for CBM

1. Refilling fuel supplied through the gas grid

2. Refilling fuel supplied by in swap bodies

$>$ Refilling methods considered for LBM

1. Refilling as the gasified liquid

2. Dual purpose refilling (gasified liquid or liquid)

Climate change impact and energy intensity parameters only reflect the operational phase since a lifecycle perspective has been purposely omitted. Nevertheless, the cost effectiveness includes both operational and investment facets which is conducive to real time decision making.

\subsection{Research}

The main method used to carry out the research has been interviewing. Data collection phase has been completed via interviewing industrial personnel who have firsthand experience within the supply chain. The people interviewed comprised of suppliers and users of biogas upgrading technologies, biomethane transportation and refilling. The suppliers of four biogas upgrading technologies and a minimum of one user of each upgrading process were interviewed concerning the biomethane production stage whereas three industrial consultants who work in biogas systems were interviewed regarding the biomethane distribution phase. Two industrial consultants and two entrepreneurs were interviewed in relation to the refilling stage. Detailed questions were presented at the interviews encompassing the $\mathrm{CO}_{2}$ and $\mathrm{CH}_{4}$ emission levels, energy 
utilization and equipment efficiencies, and investment and operational costs. The underlying intention of aforementioned interviews has been to produce tangible values for the three performance indicators which are discussed below with respect to each alternative at each stage of the supply chain. Methods used to validate data were referring to published literature and having discussions with professionals and experts of the field in tandem.

\subsection{Calculation of sustainability indicators}

In order to assess the sustainability performance of different alternatives selected under each stage of a supply chain calculations have been performed to process collected data. Consequently values were obtained with regard to the three sustainability indicators; climate change impact, energy intensity and cost effectiveness. This framework totally focuses in facilitating decision making at the operational phase of biomethane production, distribution and refilling. Therefore the indicators for the sustainability parameters were selected merely to reflect the impacts at the operational level. Table 1 elaborates how the indicators have been defined to assess the impact with regard to each aspect and which units have been used to quantify the impacts.

Table 1: Sustainability parameters and indicators

\begin{tabular}{|l|l|c|}
\hline Sustainability Parameter & \multicolumn{1}{|c|}{ Indicator } & Unit \\
\hline Climate change impact & $\begin{array}{l}\mathrm{CO}_{2} \text { equivalence of emissions } \\
\text { per unit energy of biomethane }\end{array}$ & Ton / GWh \\
\hline Energy intensity & $\begin{array}{l}\text { Energy expended per unit } \\
\text { energy of biomethane }\end{array}$ & $\mathrm{kWh}$ exp $/ \mathrm{kWh}_{\mathrm{BM}}(\%)$ \\
\hline Cost Effectiveness & $\begin{array}{l}\text { Cost per unit energy of } \\
\text { biomethane }\end{array}$ & $€ / \mathrm{MWh}$ \\
\hline
\end{tabular}

Calculation of climate change impact at the production stage assumes that the electricity used at the biomethane production facilities is produced by non-fossil fuels. Energy intensity has been measured in the amount of energy required against the energy content of biomethane. Thus it is given as a percentage. Cost effectiveness has been calculated in three elements; operational and maintenance cost, investment cost and the total cost. Also the cost effectiveness has been calculated as the cost per unit energy. The total cost figures have been used in the graphs.

The following equations have been used to calculate values for the performance factors. Investment cost effectiveness ICE ( $€ / M W h B M)$ and the Operation and maintenance cost effectiveness $O M C E$ ( $€ / M W h B M$ ) have been calculated using equations 1 and 2 :

$$
I C E=\frac{C I \times A F}{Q \times M C \times 9.67 \times 24 \times 365 \times 97 \%}
$$

$C I$ = capital investment $(€) ; A F=$ Annuity factor for the investment; $Q=$ Gas or Liquefied gas utilization rate $\left(\mathrm{Nm}^{3} / \mathrm{hr}\right) ; \quad M C=$ Methane content of the Gas/Liquid (\%); $97 \%=$ operational time.

$$
O M C E=\frac{O M}{Q \times M C \times 9.67 \times 24 \times 365 \times 97 \%}
$$

$O M=$ Operation and maintenance cost $\left(€ / \mathrm{Nm}^{3}\right)$.

Total cost effectiveness TCE ( $€ / M W h B M)$ has been calculated thus by adding specific investment cost and specific O\&M cost.

$$
T C E=I C E+O M C E
$$

Energy intensity of biomethane $E I$ (\%) has been calculated using the following equation:

$$
E I=\frac{E_{\text {in }} \times 100 \%}{Q \times M C \times 9.67 \times 24 \times 365 \times 97 \%}
$$

$$
E_{B M}=\text { energy content in biomethane (kWh) }
$$

Climate change impact of biomethane, $C I$ $\left(\mathrm{TCO}_{2 \mathrm{eq}} / \mathrm{GWh}\right.$ BM) has been calculated using the following equation:

$$
C I=\frac{C O_{2}+C O_{2 e q-B M s l i p}}{Q \times M C \times 9.37 \times 24 \times 365 \times 97 \%}
$$

\footnotetext{
1 It has been assumed that the share of cost of energy is $80 \%$ and $90 \%$ of the total $O M$ cost of CBM production technologies and LBM production technologies respectively

2 Climate impact of electricity production has been presumed zero assuming a consumption of electricity generated via non-fossil fuel sources, which is the case in Sweden.
} 
$\mathrm{CO}_{2}=\mathrm{CO}_{2}$ emission (Ton); $\mathrm{CO}_{2 \text { eq-BMslip }}=\mathrm{CO}_{2}$ equivalent of biomethane slip (Ton)

Method of costing of different biomethane distribution systems have been different considering the practical situation an investor will have to face in the context of Western Europe. For gas grid systems, investment costs include the construction of pipelines. The effects of the level of urbanization and the volume of distribution on the construction costs have been considered in the calculation. Annual operation and maintenance cost of gas grids has been assumed to be $2.5 \%$ of the total investment cost.

Investment cost of swap body based distribution has been calculated upon the requirement of the compressors to compress the gas from 4 bars to 230 bars, number of swap bodies and the trailers to carry the swap bodies. That is because in the industry the biomethane producer only owns swap bodies and trailers but hires trucks with drivers for distribution.

Furthermore it has been taken into account the fact that 1 truck can carry one swap body and the trailer attached to it can carry two swap bodies making it a distribution of three swap bodies in one trip. Also the number of trips a single truck can make in a day has been assumed to be two due to the time constraints. It is the weight limit (60 ton in Sweden) that limits the quantity transported at once [6]. Operation and maintenance cost of swap body distribution includes the fuel cost, truck hiring cost and driver's cost where the first two are driven by the distance and third driven by the time of operation.

Investment cost of distribution of liquefied biomethane depends upon the size of the trailer. A 12 ton trailer available in Sweden which has a volume of 13125 $\mathrm{Nm}^{3}$ is sufficient for the distribution of biomethane at production rates of $300 \mathrm{Nm}^{3} / \mathrm{hr}$ and $750 \mathrm{Nm}^{3} / \mathrm{hr}$ whereas for higher production rates 24 ton trailer available in Sweden which has a volume of $26250 \mathrm{Nm}^{3}$ is sufficient to meet the distribution demand. Operation and maintenance cost calculation of LBM distribution is similar to the method of swap body distribution.

\subsection{System specifications}

Table 2 contains the specifications that define the system with regard to the three stages of biomethane supply chain; production, distribution and refilling.
Table 2: Specifications of the System

\begin{tabular}{|l|l|l|}
\hline Stage & Parameter & Specification \\
\hline \multirow{2}{*}{$\begin{array}{l}\text { Biomethane } \\
\text { production }\end{array}$} & Annual operating time & $8,497 \mathrm{hr}(97 \%)$ \\
& Plant lifetime & 20 years \\
& Methane content in raw gas & $60 \%$ \\
\hline \multirow{3}{*}{$\begin{array}{l}\text { Biomethane } \\
\text { distribution }\end{array}$} & Gas grid lifetime & 30 years \\
& Swap body and trailer lifetime & 10 years \\
& Maximum weight for trucks & $60 \mathrm{~T}$ \\
& Cost of diesel & $1.52 € /$ liter \\
\hline Biomethane & Filling station lifetime & 20 years \\
Refilling & LHV of methane & $9.67 \mathrm{kWh} / \mathrm{Nm}^{3}$ \\
& Density of methane gas & $0.68 \mathrm{~kg} / \mathrm{Nm}^{3}$ \\
& Exchange rate considered & $1 €=9.0 \mathrm{SEK}$ \\
General & Electricity price for 2011 & $0.0767 € / \mathrm{kWh}$ \\
& Heating fuel price for 2011 & $0.0566 € / \mathrm{kWh}$ \\
& Discount rate & $5 \%$ \\
\hline
\end{tabular}

\section{RESULTS AND DISCUSSION}

In this section the three sustainability indicators; climate change impact, energy intensity and cost effectiveness have been discussed with respect to entire biomethane supply chains. Thus three supply chains that have the best performance pertaining to each indicator have been considered. In order to select the three supply chains it was also ensured that the best ones from each of the following systems have been selected. Thereby all the varieties of supply chains are addressed.

\section{$\checkmark \quad$ LBM system \\ $\checkmark \quad$ CBM system with swap body distribution \\ $\checkmark \quad$ CBM system with gas grid distribution}

\subsection{Climate change impact}

The climate change impact of biogas upgrading only depends on the characteristics of the technology. Among the different upgrading technologies the climate change impact is lowest in the $\mathrm{CO}_{2}$ wash process which is one of the LBM processes. It generates an impact of 0.66 tons of $\mathrm{CO}_{2}$ equivalent per $\mathrm{GWh}$ of biomethane $\left(\mathrm{TCO}_{2 \text { eq }} / \mathrm{GWh}_{\mathrm{BM}}\right)$ produced [7]. In comparing between the two CBM technologies, climate change impact in the chemical scrubbing process is $1.48 \mathrm{TCO}_{2 \text { eq }} / \mathrm{GWh}_{\mathrm{BM}}$ [8] which is approximately 10 times lower than that of the water scrubber. This difference also represents the average for amine based chemical scrubbing and water scrubbing processes at industrial scale according to Avfall Sverige [9] and Patterson et al. [10], and is totally due to the difference of the methane slip. However, the chemical scrubbing technology, CApure ${ }^{\mathrm{TM}}$, requires $0.6 \mathrm{KWh}$ of heat energy per cubic meter of raw gas of which $75 \%$ is used for pre heating 
and is normally supplied by the waste heat of the associated biogas production plant. Thereby the remaining 25\% $(0.15$ $\mathrm{KWh} / \mathrm{Nm}^{3}$ raw gas) has to be supplied from an external source of heat $^{3}$ [8]. Assuming use of non-fossil fuel (firewood is used in Sweden) the climate change impact of the use of this external fuel has been omitted in this study. However, where fossil fuel is being used, the respective climate change impact in terms of $\mathrm{CO}_{2}$ emissions has to be accounted for.

Between the alternative CBM distribution methods, operation of gas grid is by far preferred in a climate perspective as it avoids the use of fossil fuels. It had been reasonable to assume a methane slip of $0.23 \%$ of the total gas volume in pipelines in earlier times nevertheless in the context of western Europe it is even less and hence could assumed to be negligible [11]. The impact of distribution in trucks depends on both quantity distributed and the transportation distance. The larger the quantity transported, the lower the impact would be per unit of biomethane transported. Therefore, distribution of biomethane in liquefied form has a great advantage over the gaseous form since liquefaction cause the fuel to condense and thereby increase the energy density by more than 2.5 times compared to the gaseous biomethane compressed to 230 bars. Yet the longer the transportation distance the higher the impact would be, owing to the increase in $\mathrm{CO}_{2}$ emissions from the truck.

The impact of CBM refilling is 1.48 $\left(\mathrm{TCO}_{2 \text { eq }} / \mathrm{MWh}_{\mathrm{BM}}\right)$ irrespective of whether it is connected to the gas grid or delivered in swap bodies. That is because methane slip only takes place in the compressors involved in refilling. Modern storage systems are designed to recover the evaporation losses. Refilling of LBM could be considered as of zero climate change impact as the use of compressors has been avoided, and the operation of cryogenic pumps could be assumed to have negligible methane slip [12]. Figure 1 contains a comparison of the three best performing biomethane systems with a distribution distance of $10 \mathrm{~km}$, from a climate perspective.

\subsection{Energy intensity}

The least energy intensive production process for CBM would be the water scrubbing process, COMPACT ${ }^{\mathrm{TM}}$, which accounts for $3.98 \%$ of the energy produced [13]. The chemical scrubbing process, CApure ${ }^{\mathrm{TM}}$, accounts for a $4.73 \%$ utilization of the energy produced [8]. Moreover should it not receive $75 \%$ of the heating requirement from an existing waste heat source, the energy intensity would rise even higher. These results are also compatible with the figures for industrial average. The energy requirement of

\footnotetext{
3 When biogas is being upgraded elsewhere and waste heat of the biogas production plant is not obtained for pre-heating, the entire $0.6 \mathrm{KWh}$ would have to be supplied from an external source.
}

water scrubbing processes on average is $0.41 \mathrm{kWh} / \mathrm{Nm}^{3}$, which corresponds to $4.2 \%$ of the energy content of biomethane produced and the energy requirement of amine based chemical scrubbing processes on average is $0.48 \mathrm{kWh} / \mathrm{Nm}^{3}$, corresponding to $4.9 \%$ energy intensity [10]. The cryogenic process GPP ${ }^{\circledR}$ plus is the least energy intensive method to produce LBM with an energy requirement of $7.66 \%$ of the energy produced [14]. The other cryogenic process, $\mathrm{CO}_{2} \mathrm{Wash}^{\star}$, carries a substantially higher figure of $11.59 \%$ [7].

Distribution of CBM through gas grid is the most preferred method of biomethane distribution from an energy perspective. That is because the energy requirement is negligible as the distribution grids can operate at the same pressure biomethane is produced at, which is considered as 4 bars in this study. In contrast composite swap bodies need to compress the gas from 4 bars to 230 bars. That requires between $4.5 \%$ to $5.0 \%$ energy per unit of energy distributed. This significant energy usage is due to the involvement of compressors.

Energy intensity of the refilling of CBM is largely dependent on the state of incoming fuel and therefore reflects the opposite case compared to the situation in the distribution. It requires $2.67 \%$ of the amount of energy refilled to compress the biomethane received in gas grids, from 4 bars to 230 bars whereas refilling biomethane in swap bodies which is already above 230 bars, requires $0.75 \%$ [12].

Figure 2 depicts the different performance levels of alternative biomethane systems based on their energy intensities. The distribution distance considered is $10 \mathrm{~km}$. It is thereby clear that operating a gas grid to distribute biomethane is a preferred alternative over composite swap bodies in a CBM system from an energy perspective. Also adopting a CBM system in general is desirable over an LBM system which is highly energy intensive especially during production. 


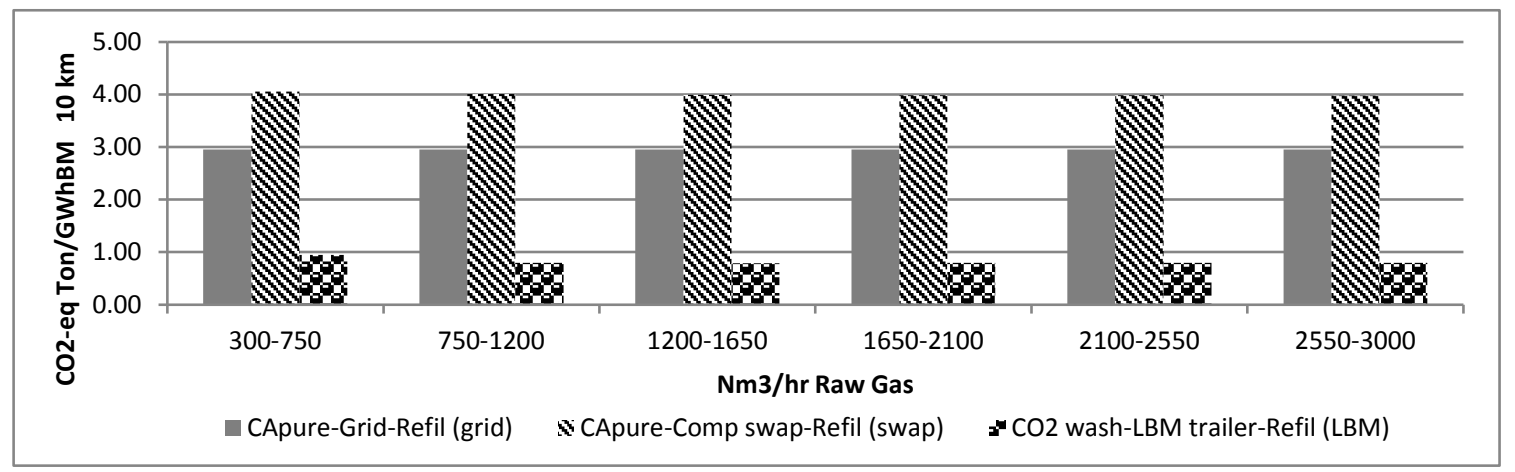

Figure 1: Climate change impact of Alternative Biomethane Systems at 10km Distribution

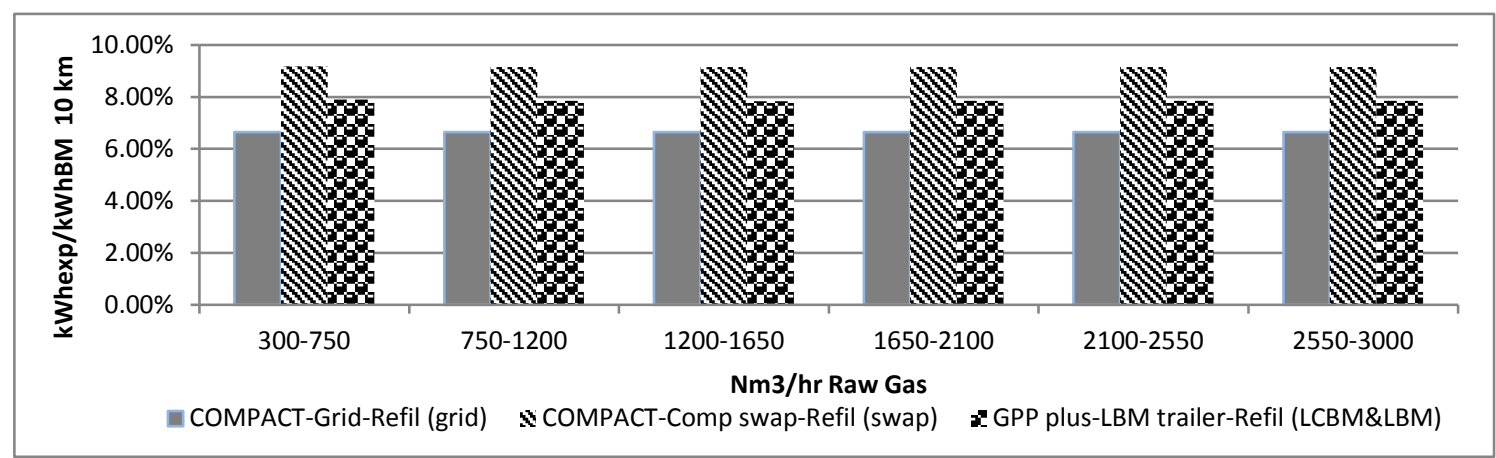

Figure 2: Energy intensity of Alternative Biomethane Systems at 10km Distribution

\subsection{Cost effectiveness}

In general, production of LBM is much costlier than the production of CBM owing to the substantial investment and operational costs associated with the cryogenic technologies. The most cost effective means of upgrading biogas is the chemical scrubbing process, CApure ${ }^{\mathrm{TM}}$, where the total cost effectiveness ranges from $8.63 € / \mathrm{MWh}_{\mathrm{BM}}$ at $300-750 \mathrm{Nm}^{3} / \mathrm{hr}$ production level to $5.29 € / \mathrm{MWh}_{\text {BM }}$ at 2550-3000 Nm $3 /$ hr production level [8]. In LBM production, GPP $p$ lus process is the most cost effective at all production levels.

The cost effectiveness of biomethane distribution substantially depends on the quantity distributed and the distance of distribution. In the distribution of CBM, gas grids are special due to the large dependence of its investment cost on the distance of distribution while almost being independent of the quantity distributed. However, operation and maintenance cost of a gas grid can assumed to be only dependent on the distribution distance but independent of the quantity distributed. In contrast, distribution in trucks has a fixed investment cost irrespective of the distance of distribution whereas the operation and maintenance cost is directly related to both distance and quantity. On the other hand distribution of biomethane in the liquefied form using LBM trucks has promising cost effectiveness as opposed to both CBM distribution methods in most of the cases, except for short distance distribution of large quantities. That is solely due to the increased density of methane in liquefied form which leads to less space requirement. Thereby it is able to transport a 2.5 times more volume than in the composite swap bodies [15]. In short distance $(10 \mathrm{~km})$ biomethane distribution, total cost effectiveness (TCE) of gas grid is better than composite swap bodies at all distribution capacities considered. For example, the TCE of gas grid and composite swap bodies are $11.84 € / \mathrm{MWh}_{\text {BM }}$ and 12.37 $€ / \mathrm{MWh}_{\text {вм }}$ respectively at the distribution capacity of 300 $750 \mathrm{Nm}^{3} / \mathrm{hr}$ which is the lowest level considered in the study. The same figures for the distribution capacity of $2550-3000 \mathrm{Nm}^{3} / \mathrm{hr}$ (which is the highest level considered in the study) are $2.16 € / \mathrm{MWh}_{\mathrm{BM}}$ and $8.07 € / \mathrm{MWh}_{\mathrm{BM}}$. This situation slightly changes once we move into the medium distance distribution level $(25 \mathrm{~km})$. Truck distribution has a better TCE of $14.13 € / \mathrm{MWh}_{\mathrm{BM}}$ as opposed to the gas grid with a TCE of $18.94 € / \mathrm{MWh}_{\mathrm{BM}}$ at the lowest capacity level $\left(300-750 \mathrm{Nm}^{3} / \mathrm{hr}\right)$. Subsequently, the gas grid takes over and shows comparatively a better performance as the distribution capacity increases. When it comes to long distance distribution $(100 \mathrm{~km})$ gas grid has a poor TCE against truck distribution owing to the very high investment costs involved, even though the operation and maintenance costs are less in gas grids. Distribution of biomethane in the liquefied form in LBM trucks is the cheapest of all methods as far as the biomethane production levels considered in this study are concerned. 
The cost of refilling CBM again depends on whether the filling station receives fuel through the gas grid or in swap bodies. Refilling CBM from swap bodies is much cheaper than refilling from the gas grid. That is merely because of the requirement to compress the fuel in gas grid from 4 to 230 bars, in contrast to the fuel in swap bodies which is already compressed to 230 bars (compressing cost associated with swap bodies have been taken into account at the distribution stage). Refilling in the liquefied form is the cheapest among all because it eliminates the requirement of compressors of which the investment and operational cost are very high.

The costs are scattered in different sectors of the supply chain from upgrading to refilling. This has made it difficult to distinguish between the performances of different biomethane routes available by looking into the situation at each stage. Thus adopting a systems perspective will foster a holistic comparison of alternative biomethane supply chains. Thereby it avoids the possibilities of generating different interpretations based on the performance levels at individual stages of the supply chain.

Deciding between CBM systems based on the cost factor is always a matter of the specific situation. According to figure 3 , if the distribution distance is short $(10 \mathrm{~km})$ the CBM systems based on a grid distribution will have a better cost performance over the CBM systems based truck transportation at all production levels except for the lowest level $\left(300-750 \mathrm{Nm}^{3} / \mathrm{hr}\right)$. As the distribution distance increases, the CBM system based on gas grid gradually gives away the better cost performance it enjoyed at hire production levels, to the CBM system based on truck transportation using swap bodies. That is because of the burden of capital investment in constructing gas grids compared to the cost of hiring trucks. Figure 4 shows how CBM systems based on distribution in composite swap bodies catch up with the systems based on gas grids at the production rate $1200-1650 \mathrm{Nm}^{3} / \mathrm{hr}$ at medium distance (25 $\mathrm{km}$ ) distribution scenarios. As it is shown in figure 5 adopting CBM systems based on swap body distribution is always better than systems based on gas grids in the perspective of total cost effectiveness.
Adopting an LBM system becomes less cost effective compared to a CBM system at two specific instances. That is only when it is short distance $(10 \mathrm{~km})$ distribution and at production rates of $2100-2550 \mathrm{Nm}^{3} / \mathrm{hr}$ and $2550-3000 \mathrm{Nm}^{3} / \mathrm{hr}$. Despite that the cost effectiveness of the LBM system over CBM system remains unaffected irrespective of the distribution length or the level of biomethane production.

\subsection{The benchmarking framework}

The framework used in this article specifically benchmarks biomethane supply chains via identifying the best practices in production, distribution and refilling of biomethane. Therefore this framework as a whole cannot be used for evaluations of alternative fuel supply chains For example; biogas and petroleum. However, natural gas systems can be put into the distribution and refilling stages for benchmarking as the content and the composition of natural gas is very much similar to biomethane and can be delivered and refilled exactly the same ways as biomethane. But the production of natural gas cannot be put into this framework as the production phase of this framework particularly deals with upgrading process of biogas.

According to the University of Southern Queensland a benchmarking framework should be able to be used in a continuous process where comparative evaluations used for improvements [16]. Thus, in order to accord with a continuous improvement approach, the framework presented here has to be updated on a regular basis along with the development of the technologies in the biomethane supply chain. The evaluation process of sustainability parameters are already in a ready-to-update format however, more improvements are required in terms of accessibility compared to many other benchmarking frameworks which are readily accessible, specifically though the internet.

Developing a readily updatable model of this framework and incorporating it to a web-based platform can therefore be the future work associated with this research. 


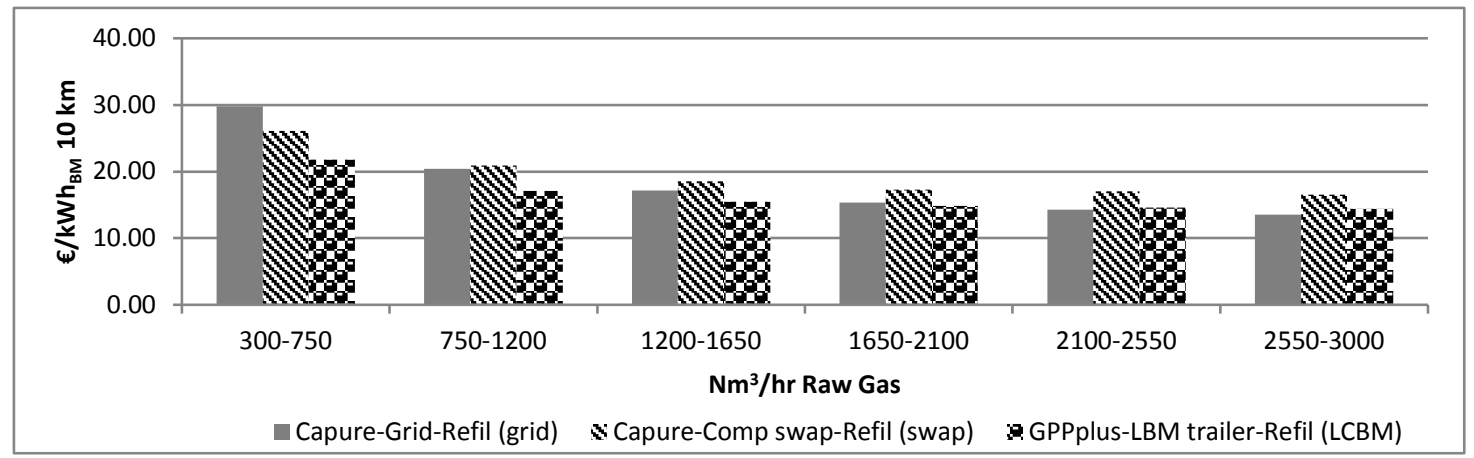

Figure 3: Cost Effectiveness of Alternative Biomethane Systems at 10km Distribution

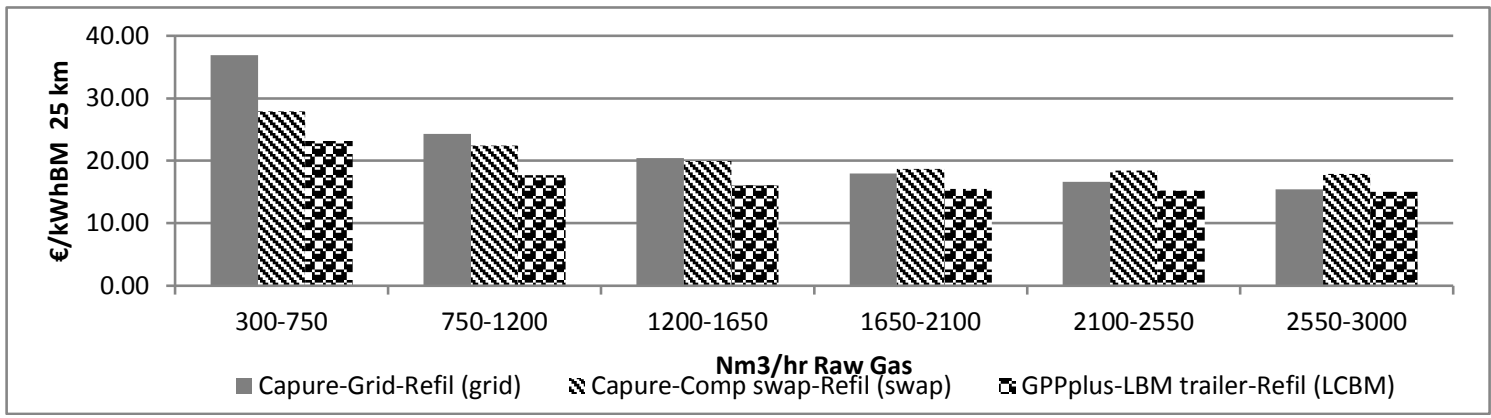

Figure 4: Cost Effectiveness of Alternative Biomethane Systems at 25km Distribution

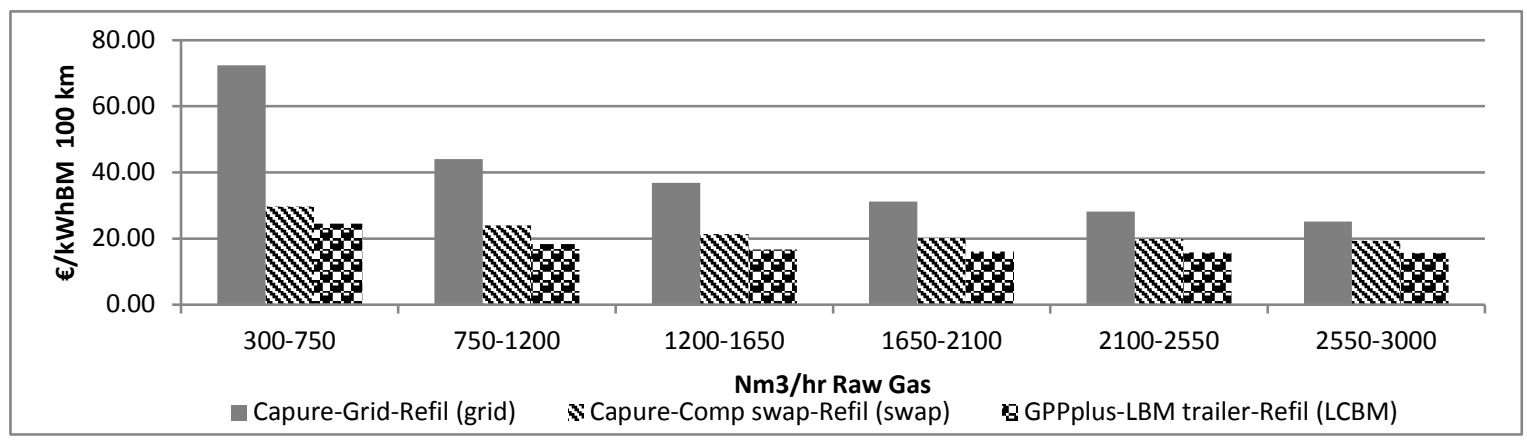

Figure 5: Cost Effectiveness of Alternative Biomethane Systems at $100 \mathrm{~km}$ Distribution

\section{SENSITIVITIES OF RESULTS}

Following are the crucial aspects identified in this study and the effects of their sensitivities to the results of this study.

\subsection{Raw gas quality}

This is a serious factor that can affect both the energy intensity and cost effectiveness of an upgrading plant. Normally if the incoming biogas quality varies from the design level it is kept circulating for some time without being introduced into the process in order to homogenize. This practice dramatically increases the energy intensity and operational cost of the plant. Therefore the fact that how fast a plant can adapt to the varying raw biogas quality is a deterministic factor of those two indicators [17].

\subsubsection{Fuel price}

Cost of distribution in trucks is prone to fluctuations in fuel (diesel) prices. Any change in fuel price will have an instant and direct impact on the cost of operation of biomethane distribution in swap bodies and in LBM trailers. Therefore it has been vital to analyze the sensitivity of cost effectiveness of distribution systems to changing fuel prices, which might have a significant influence over the conclusions of the study. Since distribution in gas grid does not require a significant energy to transport CBM, it would be momentous to look upon the relative performance of the gas grid to the distribution in trucks, for changes in fuel price. Thus a $50 \%$ fuel price increase has been considered which would be the maximum justifiable in the foreseeable future. 
The maximum effect of the fuel cost on distribution in composite swap bodies could be seen over the maximum distribution distance which is $100 \mathrm{~km}$. Yet the fuel price is a relatively small element that ranges between $10 \%-13 \%$ (depending on the quantity distributed) of the total distribution cost while investment cost (50\% - 30\%) and truck hiring cost $(24 \%-30 \%)$ being the major contributors. Other elements of distribution cost are; compressing cost $(11 \%-19 \%)$ and swap body handling cost (5\% - 8\%). The percentages are given respectively for biomethane production rates of $300 \mathrm{Nm}^{3} / \mathrm{hr}$ and $3,000 \mathrm{Nm}^{3} / \mathrm{h}$. Therefore even a $50 \%$ increase in the fuel price would only cause $5 \%$ $6 \%$ increase in the total cost of biomethane distribution in composite swap bodies. This cost increase is not sufficient to cause a noticeable shift in the relative economic attractiveness of distribution in composite swap bodies against distribution in gas grids at any capacity $\left(300 \mathrm{Nm}^{3} / \mathrm{hr}\right.$ $\left.-3,000 \mathrm{Nm}^{3} / \mathrm{h}\right)$, and over any distance $(10 \mathrm{~km}-100 \mathrm{~km})$. The case of distributing LBM using trucks is therefore quite clear as it is even cheaper than composite swap bodies, since it can carry larger quantities of biomethane in a single load.

Thus the cost effectiveness of biomethane distribution was recognized as insensitive to the fuel price.

\subsubsection{Transportation weight limit}

Some countries in the Western Europe such as Norway have a maximum weight limit for road transportation of fuel of 50 ton in contrast to 60 ton in Sweden [15]. It thus affects the number of journeys required to transport the fuel. Thus the operational cost of distribution increases as the maximum weight limit decreases. Therefore in contrast to the fuel price which has a direct impact on operational cost, weight limit which has an indirect impact causes much deviation in the total cost effectiveness of distribution since it affects the number of journeys a truck has to make, which in turn causes all the elements of operational cost to change.

\subsubsection{Installed capacity}

As the trend shows in figure 5, distribution of CBM in gas grid would be more cost effective than swap bodies at extended production rates. Thus figure 6 elaborates the cost effectiveness of the two CBM distribution methods between the distance levels $25 \mathrm{~km}$ and $100 \mathrm{~km}$ at the next level of biomethane production further to the maximum considered in this study, which is $3,450 \mathrm{Nm}^{3} / \mathrm{hr}$ RG. Thereby it is evident that distributing CBM in gas grids between 25 $\mathrm{km}$ to $100 \mathrm{~km}$, which has been previously uneconomical, compared to distribution in composite swap bodies at a $3,000 \mathrm{Nm}^{3} / \mathrm{hr}$ production level, is now economical up to 40 $\mathrm{km}$ distribution. Economic performance of gas grids would be even better at higher levels of biomethane distribution.

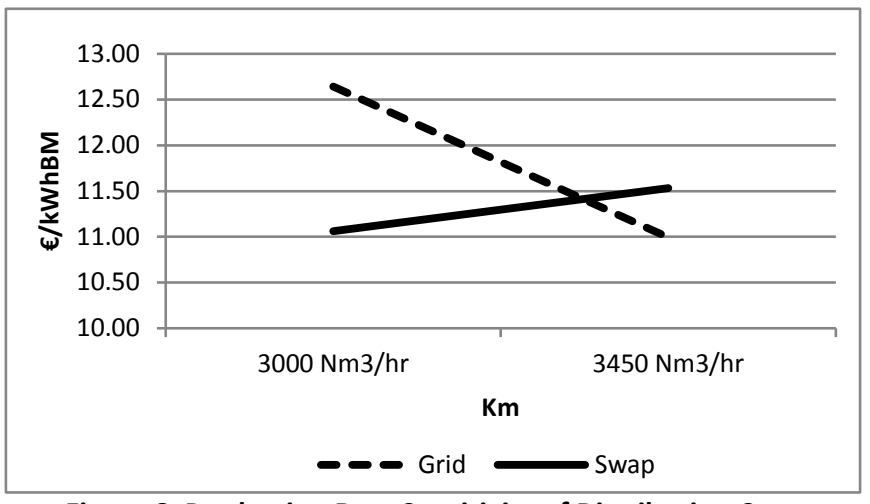

Figure 6: Production Rate Sensitivity of Distribution Cost Effectiveness

\section{CONCLUSIONS}

The systems analysis on biomethane supply chains with regard to sustainable performance has paved the way to several vivid conclusions.

Adopting an LBM system is preferred over a CBM system from both climate and cost perspectives at all levels of biomethane production whereas LBM supply chain lags behind from an energy perspective due to the very high energy intensity during the production phase.

Supply chains consisting of gas grid distribution is only economical either at very large scale production or at short distance distribution despite its superior performance in climate and energy aspects. That is solely owing to the dramatic cost of investment associated with construction. A combination of both high rate production and short distance distribution will therefore be ideal for fostering gas grid based CBM systems. Nevertheless, existing gas grids should be used as much as possible because gas grids are economically unattractive only in the case of a brand new investment. A profound alternative would be to form joint ventures into gas grids by which several suppliers can collectively deliver a large quantity of gas and distribute cost effectively through longer distances. Thereby it will become a very sustainable alternative since gas grids neither cause adverse climate change impact nor high energy intensity. In other cases, distribution in composite swap bodies may replace the use of gas grids which will however be a compromise of environmental health over economic interests.

Several factors of which, the fluctuations can affect the accuracy of results, are also identified. Even though fuel price could increase significantly and instantly, it does not cause a noticeable shift in the relative cost performance of alternative distribution methods. In contrast, the distribution cost effectiveness is sensitive to the variations in maximum transportation weight limit on roads. As it has been identified the cost performance of distribution is also sensitive to any extensions of biomethane production rates that can be made further to the maximum considered in this study. 
Supply of liquefied biomethane gives the most promising results based on climate performance whereas the energy intensity is fairly high. The economic performance of LBM systems is also the best, especially in long distance distribution. The production technologies of liquefied biomethane however are still in their maturing stages and hence a considerable possibility exists that gaps in the consistency of alleged performance levels by the suppliers could be expected in reality. Thus the supply chain analyses are prone to the accuracy of the information obtained from the suppliers of the two cryogenic technologies. Hence it is recommended that project implementation should follow detailed technical studies specifically in the context of the situation with the assistance of the results of this article.

\section{REFERENCES}

\subsection{Citation in text}

[1] European Commission Eurostat. 2011. Transport energy consumption and emissions, 2004.

http://epp.eurostat.ec.europa.eu/statistics_explained/inde x.php?title=File:Transport_energy_consumption_and_emiss ions,_2004.PNG\&filetimestamp=20090430100043.

Accessed 5 June 2011

[2] Malmberg, 2014. Reliability and Easy maintenance with Malmberg COMPACT ${ }^{\circledR}$. (Translated from Swedish to English).

http://www.malmberg.se/biogas/malmberg_compact_1

Accessed 3 March 2014

[3] Purac Puregas, 2014. The CApure ${ }^{\mathrm{TM}}$ process.

http://purac-puregas.com/?page_id=58

Accessed 3 March 2014

[4] GTS Gastreatment Services BV, 2014. Technology.

http://www.gastreatmentservices.com/en/?option=com_co ntent\&view=article\&id=33\&ltemid=46\&lang=en

Accessed 3 March 2014

[5] Terracastus technologies, 2014. Technology for clean and upgrading biogas

http://www.businessregion.se/download/18.3b74207e11ca cd3cffe80008761/Production+of+liquified+biomethane+Ter racastus+Technology_Johan+Beyer+\&+Bill+Brown.pdf Accessed 3 March 2014

[6] Benjaminsson, J. 2011., Swedish Gas Center AB, Malmö, Sweden, Personal communication.

[7] Salm, K.V. D. 2011., Teracastus Technologies AB, Gothenburg, Sweden, Personal communications.

[8] Karlsson, L.E. 2011., Biogas Upgrading Division, Läckeby Water Group, Kalmar, Sweden, Personal communication.
[9] Avfall Sverige. 2008. Frivilligt åtagande Kartläggning av metanförluster från biogasanläggningar 2007-2008 (Voluntary Commitment Mapping of methane losses from biogas plants 2007-2008). Development/2009U, Malmö: Avfall Sverige AB.

[10] Patterson, T. Esteves, S. Dinsdale, R. Guwy, A. 2011. An evaluation of the policy and techno-economic factors affecting the potential for biogas upgrading for transport fuel use in the UK. Energy policy. 39 (3): 1806-1816.

[11] Pertl, A. Mostbauer, P. Oberstainer, G. 2010. Climate balance of biogas upgrading systems. Waste management. 30 (1): 92-99.

[12] Henrik, N. 2011., Cryo AB, Gothenburg, Sweden, Personal communication.

[13] Näslund, D. 2011., Näslund, Malmberg Water AB, Åhus, Sweden, Personal communication.

[14] Kramer, S. 2011., Gastreatment Services BV, Rotterdam, Netherlands, Personal communication.

[15] Benjaminsson, J. Nilsson, R. 2009. Distributionsformer för biogas och naturgas i Sverige (Distribution of biogas and natural gas in Sweden). Stockholm: Grontmj AB.

[16] USQ (University of Southern Queensland), 2008. A Benchmarking Framework for the University of Southern Queensland. Huston: American Productivity and Quality Cente.

[17] Schmuderer, M., 2010. Overview, operational experience and perspectives of biogas upgrading technologies. Paper presented at international conference on the road with CNG and Biomethane, 4-5 February, Prague.

\section{ACKNOWLEDGEMENT}

Authors' sincere acknowledgment is bestowed upon all the resource persons for their generous cooperation towards sharing the knowledge, experience and facts. It was very necessary to produce a research article such as this one which deals more with the pragmatic and applied engineering issues in the real-time context. 